\title{
Dislocation-obstacle interactions: dynamic experiments to continuum modeling
}

\author{
I. M. Robertson, ${ }^{1}$ A. Beaudoin, ${ }^{2}$ K. Al-Fadhalah, ${ }^{2}$ Chun-Ming Li, ${ }_{3}^{1}$ J. Robach, ${ }^{1}$ B. D. Wirth, ${ }^{4}$ A. \\ Arsenlis, ${ }^{5}$ D. Ahn, ${ }^{3}$ and P. Sofronis. ${ }^{3}$ \\ ${ }^{1}$ Department of Materials Science and Engineering, ${ }^{2}$ Department of Mechanical and Industrial \\ Engineering, ${ }^{3}$ Department of Theoretical and Applied Mechanics, University of Illinois \\ Urbana, IL; ${ }^{4}$ Department of Nuclear Engineering, University of California- Berkeley, \\ ${ }^{5}$ Lawrence Livermore National laboratory, Livermore, CA.
}

\begin{abstract}
Incorporating the interaction of dislocations with obstacles remains a challenge in the development of predictive large-scale plasticity models. The need is particularly important in the elastic-plastic transition region where these interactions can dominate the behavior. By combining post-mortem analysis with dynamic straining in the transmission electron microscope, the atomic processes governing glissile dislocation reactions and interactions with obstacles has been determined. This information has been incorporated at least phenomenologically in models to assess the macroscopic stress-strain response. Two examples will be presented to demonstrate the methodology. The first example considers the interaction of dislocations with small vacancy Frank loops and the formation of defect-free channels in copper, and the second with the influence of imperfect annealing twin boundaries on the macroscopic stress-strain response in silver. In both examples, the importance of grain and twin boundaries as dislocation sources will be demonstrated.
\end{abstract}

\section{Introduction}

Dislocations and their interactions with other microstructural features, such as impurity atoms, precipitates, dispersoids, dislocations, and grain boundaries must be incorporated into the constitutive relationships that describe plastic deformation of metallic systems. However, despite considerable progress, no clear methodology exists for transferring this information into a predictive macroscopic constitutive relationship. ${ }^{[1-7]}$

In this paper, we present two examples to illustrate how conventional static transmission electron microscopy and in-situ straining in the transmission electron microscope can be combined to understand microstructural evolution during straining and how this information can be incorporated into plasticity models. The importance of grain and twin boundaries as sources of dislocations is demonstrated. The first case considers the interaction of lattice dislocations with small Frank loops in ion irradiated copper ${ }^{[6,8]}$ and the second the impact of imperfect annealing twins in fine grained silver on the macroscopic stress strain response. ${ }^{[9]}$

\section{Experimental Procedure}

To investigate the interaction of dislocations with Frank vacancy loops, in situ straining samples (dimensions $11 \times 2.7 \times 0.25 \mathrm{~mm}$ ) were cut from $99.999 \%$ pure polycrystalline $\mathrm{Cu}$. Following annealing, the central section was thinned to electron transparency by jet electropolishing in 
a solution of $33 \%$ nitric acid in methanol cooled to $-208{ }^{\circ} \mathrm{C}$ at a current density of

$0.11 \mathrm{~A} \mathrm{~cm}^{-2}$. These electron transparent samples were irradiated with $200 \mathrm{keV} \mathrm{Kr}^{+}$ions to a dose between $1 \times 10^{11}$ and $1 \times 10^{13}$ ions $\mathrm{cm}^{-2}$ at room temperature at Argonne National Laboratory ${ }^{[10]}$. This irradiation created small vacancy type Frank loops throughout the thinned foil.

The silver used in this study was received after having been severely cold rolled and annealed and then deformed in tension at room temperature at a strain rate of $10^{-3} \mathrm{sec}^{[11]}$ In addition, some tests were interrupted at strains of 0.001 and $0.01 \%$. These samples provided insight to the microstructure and were used in the in situ study to determine the processes responsible for generating the microstructure. To produce transparent specimens for TEM examination, bulk samples were cut, ground, and finally punched to $3 \mathrm{~mm}$ diameter discs with a thickness of $120 \mu \mathrm{m}$. The samples were jet-electropolished to perforation in a solution of glacial acetic acid, concentrated sulphuric acid, anhydrous methyl alcohol, and thiourea at 5 volts and at $10^{\circ} \mathrm{C} .^{[12]}$ These $3-\mathrm{mm}$ discs were glued to a template that could be used in the straining stage.

The in situ straining experiments were performed at room temperature in a JEOL 4000 EX electron microscope equipped with a TV-rate camera system. The dynamic events were recorded on S-VHS for later analysis.

\section{Experimental Results and Discussion \\ Case1. Dislocation interactions with Frank loops}

The macroscopic stress-strain response of irradiated materials show the general behavior of a loss of ductility, an increase in yield and tensile strength, and the appearance of an upper yield point, even in FCC materials, at high defect densities. ${ }^{[13-20]}$ Microstructurally, these property changes must be correlated with channels from which the defects have been annihilated. ${ }^{[21,22]}$ Understanding the origin of the dislocations that create the channels, the process(es) by which the dislocation-defect interaction annihilates the defect, the mechanisms controlling the width of the channel is needed to allow development of a large-scale predictive model. In this paper the emphasis is on the origin of the channels, specific annihilation mechanisms are not considered in this paper.

It has generally been assumed that the pre-existing lattice dislocations are trapped by a "Cottrell-like" atmosphere of small interstitial clusters - this is referred to as the cascade-induced source hardening model. These clusters are created within the displacement cascade and glide to the dislocation where they are trapped. ${ }^{[23-28]}$ Thus, the increase in the yield strength and the appearance of the yield point drop at high defect densities (high irradiation doses) is attributed to segments of the pre-existing dislocations breaking free from the atmosphere to create a dislocation source. ${ }^{[27,28]}$ The source then generates the dislocations of the same type needed to create the channel. A dispersed-arrier hardening model is then introduced to account for the effect of the dislocations moving through a field of obstacles. In the following sections, these assumptions are brought into question and it is shown that the macroscopic stress-strain curve can be accounted for within the framework of a dispersed-barrier hardening model; a more detailed account can be found in Robach et al. ${ }^{[6]}$ 


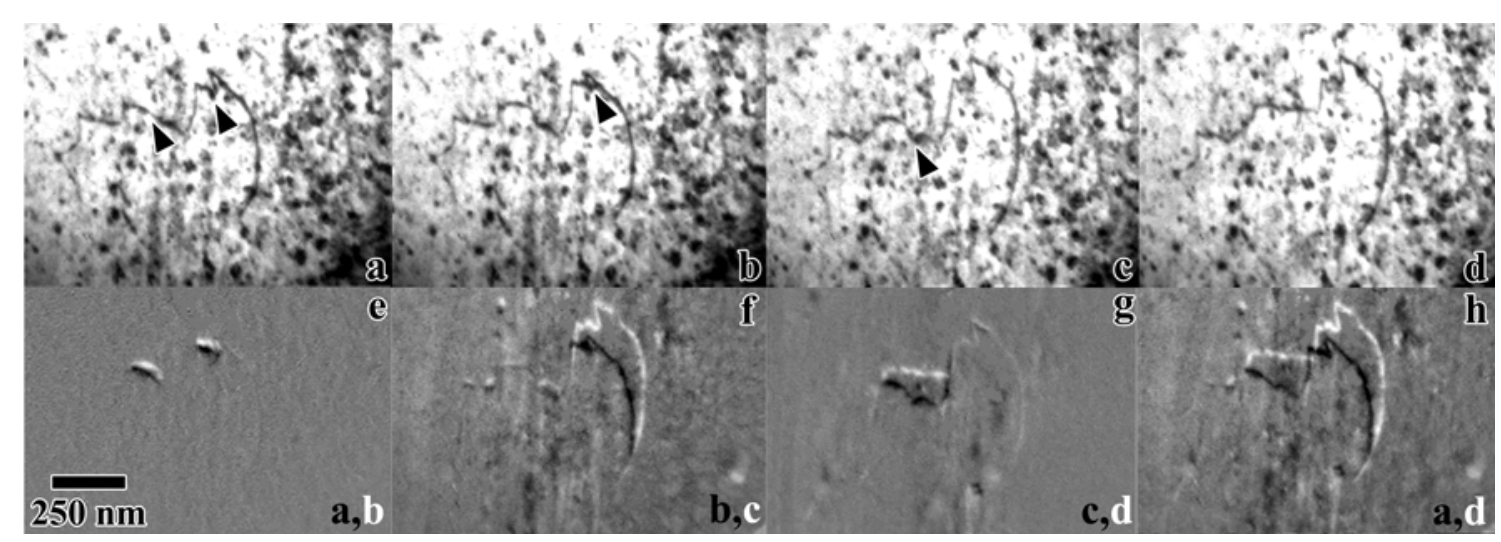

Figure 1. Bright-field images showing the percolation of pre-existing dislocations through the defect field, (a)-(d). Comparison images with the white dislocation showing the dislocation position at the later time, (e)-(h). Arrowheads indicate pinned points about to break free in the next frame. ${ }^{[6]}$

The electron transparent ion irradiated samples were loaded in tension in the transmission electron microscope at room temperature. The pre-existing dislocations were largely immobile although some motion was observed. Evidence for this is presented in Fig. 1 in which the motion of an isolated pre-existing dislocation as a function of increasing strain is shown. To accentuate the change in position comparison images, in which a negative image of a later time is superimposed on a positive image of an earlier time, are also presented. Comparison of the final (fig. 1d) and initial (fig. 1a) position of the dislocation, fig $1 \mathrm{~h}$, shows that it is not very mobile and did not form a dislocation source as required in current models. ${ }^{[27,28]}$ Analysis of the effectiveness of dislocations at annihilating defects showed that dislocations with a large screw component are more effective than ones with a large edge component. Therefore, formation of a dislocation source from a pre-existing dislocation would be difficult as expansion will occur at different rates for edge and screw segments of the loop.

The dislocations responsible for creating the channels originate from grain boundaries or stress concentrators such as a crack tip. An example of a channel originating ahead of a propagating crack is shown in Fig. 2. The first seven screw dislocations responsible for clearing the channel are indicated. The dislocations do not appear to be on exactly the same slip plane, suggesting that either multiple sources operate at the crack tip or that cross slip occurs readily in the vicinity of the crack. This may have important consequences for determining the width of the channel.

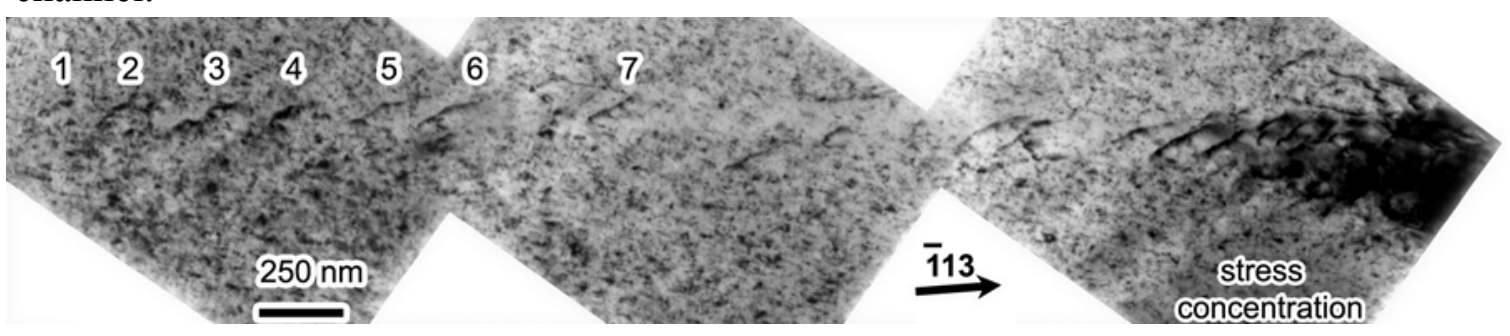

Figure 2. Dislocation emission from a propagating crack and the formation of a defectfree channel. The first seven crew dislocations are seen. ${ }^{[6]}$ 

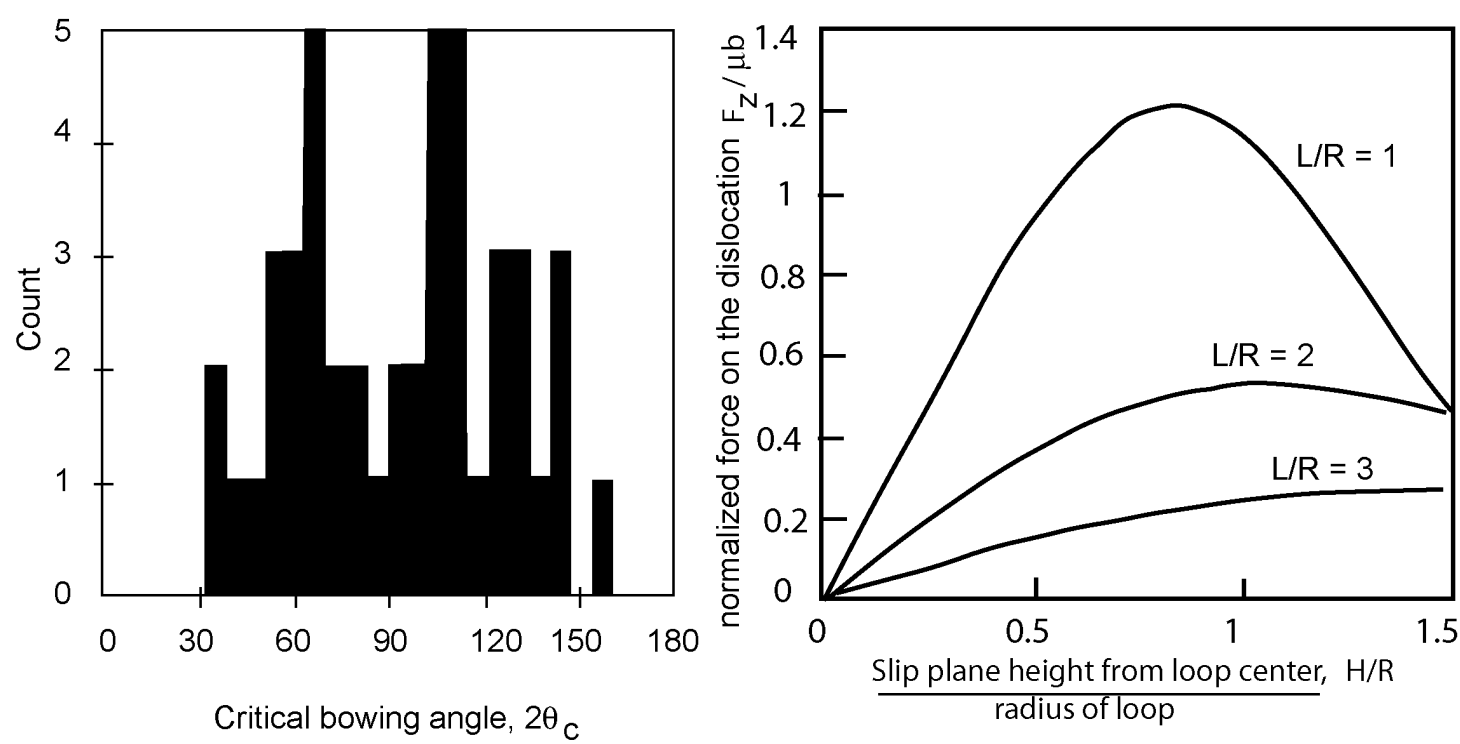

Figure 3. a) Critical bowing angle for dislocation interactions with Frank loops in Copper $^{[6]}$. b) normalized force on the dislocation due to the presence of the defect as a function of the dislocation position with respect to the loop. $\mathrm{L}$ is the distance from the loop to the dislocation, $\mathrm{R}$ is the loop radius and $\mathrm{H}$ is the distance from the loop center to the slip plane.

The in situ straining experiments permit the critical angle at which the dislocation breaksfree of the obstacle to be determined. Obstacle strengths for Frank loops are shown as the critical angle for breakaway in Fig. 3a. By using the dispersed barrier hardening theory, which incorporates the line tension of a dislocation bowing between pinning points, it is possible to obtain an estimate of the shear stress needed for the dislocations to break free of the obstacle. For these Frank loops, the barrier strength varies from 10.3 to $157.5 \mathrm{MPa}$ with an average of 49.1 MPa.

What is interesting is that for this, and other, obstacles, the obstacle strength has a range of values as opposed to the single value assumed in previous models. ${ }^{[29]}$ The range of values can be understood by considering the Peach-Koehler force between an edge dislocation and a vacancy loop for various geometries. The results of this calculation are summarized in fig. $3 \mathrm{~b}$. The change in repulsive force, $\mathrm{F}_{\mathrm{z}}$, with distance from the obstacle center, $\mathrm{H}$, is plotted for three dislocation obstacle separation distances, L. The curves show that there is a marked difference in the interaction force when the dislocation intersects the loop center $(H / R \sim 0)$ or just grazes its edge $(H / R \sim 1)$. The increase in maximum repulsive force with decreasing separation distance is expected from the Peach-Koehler equations. ${ }^{[30]}$ These calculations support the notion that the orientation and geometry of the dislocation with respect to the loop and irradiation-induced defect affects the forces involved during an interaction. This effect, coupled with the range of loop diameters that exists ${ }^{[31]}$, accounts for the variation in the obstacle strength determined experimentally, Fig. 3a.

To summarize, the dynamic microscopy study revealed that pre-existing dislocations are not responsible for creating the defect-free channels and that the dislocations are ejected from grain boundary sources and from stress concentrators; that interaction with one dislocation is not 
always sufficient to annihilate the defect; that screw dislocations are more effective at annihilating a defect than an edge; and that the obstacle strength is not a constant but depends on the obstacle size and the interaction point. The increase in the yield strength with increasing irradiation dose can be attributed to the increased difficulty of moving a dislocation from the source through the obstacle field. That is, the problem is one of propagation not nucleation.

To model the macroscopic stress-strain curve, dispersion hardening has been incorporated into an isotropic plasticity model; the details of the model can be found in Robach. [6] This model assumes that the forest dislocations and irradiation-induced defects act as obstacles and that the strength of the obstacles is not constant initially and changes with strain. To introduce the variable obstacle strength found experimentally, a distribution of obstacle sizes was introduced and the effective strength related directly to defect size. The total volume fraction of defects is assumed constant.

The irradiation-induced defects are treated as shearable obstacles that vary in size and density with plastic strain. The interaction mechanism is assumed to be by shearing. This has been observed experimentally for stacking-fault tetrahedra in low stacking-fault energy materials $^{[29]}$ and by molecular dynamics computer simulation. ${ }^{[32]}$ The line dislocation density also evolves with plastic strain according to equations developed for single crystals, see Arsenlis et al. ${ }^{[33]}$

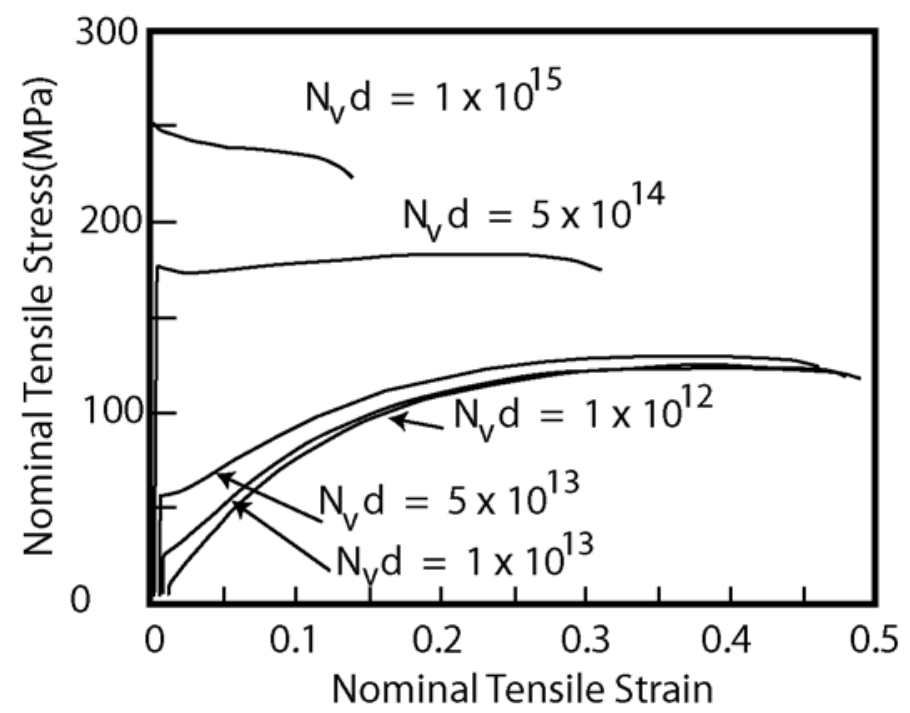

Figure 4. Stress-strain curve predicted from the model as a function of increasing defect density (irradiation dose). ${ }^{[33]}$

In a pure material, the total resistance to dislocation motion is proportional to the sum of the obstacle densities rather than the sum of individual contributions. That is, the total resistance is proportional to $\mu b\left(\alpha \rho_{\text {disl }}+\beta_{\text {def }}\right)^{1 / 2}$ where $\mu$ is the shear modulus; $b$ is the magnitude of the Burgers vector of the dislocation; $\rho_{\text {disl. }}$. and $\rho_{\text {def. }}$ are the dislocation and defect density respectively; $\beta$, is the strength of the radiation defects, and $\alpha$ is the strength associated with the forest dislocation density. For the current case, the defects are initially more effective obstacles than other dislocations, i.e., $\alpha>\beta$, although this changes at higher strains. To determine how the response depends on the irradiation dose, $\rho_{\text {def }}$ was varied while all other internal state variables were kept constant. The strain rate used in the simulations was $3 \times 10^{-4} \mathrm{~s}^{-1}$ and the initial defect size was $2.5 \mathrm{~nm}^{[33]}$ As $\rho_{\text {def. }}$ increases, the yield point increases and the initial strain hardening decreases. Constructing the total resistance as the sum of the contributions from the dislocation and defect densities reduces the rate of strain hardening in metals with increasing defect density, as shown in fig. 4. The curves in Fig. 4 are the results of full three-dimensional finite-element simulations coupled to the plasticity model and a von Mises failure criterion to produce failure. ${ }^{[33]}$ As shown in Fig. 4, at high defect densities the crystal may work soften instead of work harden because the mobile dislocation density increases faster than the dislocation mobility decreases due to the evolving forest density. The strain softening is 
due to the initial avalanche of mobile dislocations and to a reduction in the resistance due to the shearing of the defects. The initial avalanche of dislocations increases the density of both mobile and forest dislocations, although the evolving dislocation density does not change the strength of highly irradiated material as much as in an unirradiated metal. However, the multiplication of the mobile dislocations enables each gliding dislocation to move across a smaller area per unit time and thus encounter fewer forest obstacles. As a result, the strain hardening decreases monotonically with increasing irradiation-induced defect density. At larger strains, the dislocation density increases such that contributions to the resistance by the forest dislocation density and the defect density become comparable, and the metal begins to strain harden again. The softening mechanism due to the irradiation-induced defects assumes that the change in the resistance of these defects depends strongly on their pinning strength, which is determined by their size. The irradiation-induced defects are sheared by the passage of the glide dislocations such that the effective obstacle strength changes while their volume fraction remains constant during deformation. The cutting of the defects increases their density while reducing their average size, resulting in a decrease in the material resistance to further dislocation motion. These mechanisms combine and lead to the observed plastic instability while decreasing the work hardening without softening at lower defect densities. This example demonstrates the value of using microstructural characterization techniques to reveal the key features that must be included in a continuum-level model for it to yield the macroscopic stress-strain behavior.

\section{Case 2. Impact of annealing twins on the macroscopic stress-strain response of fine- grained silver.}

Prior to the macroscopic uniaxial tension tests the material had been cold-rolled and then annealed to produce annealing twins within grains with an average diameter of $2 \mu \mathrm{m} .^{[1,11]}$ The resulting stress-strain curve is shown in Fig. 5a. The tensile properties are characterized by a relatively low proportional limit (on the order of $20 \mathrm{MPa}$ ), followed by an elongated yield point at $140 \mathrm{MPa}$, and finally the usual stage III hardening behavior beyond a strain of a few percent. The challenge is to understand the evolution of plasticity in this material. To understand the microstructural evolution with strain some tensile tests were interrupted at strains of 0.001 and $0.01 \%$ and the microstructure assessed by TEM. Additional information can be found in ref. ${ }^{[9]}$

Following the anneal and prior to quasi-static testing at room temperature the microstructure was dominated by annealing twins within the $2 \mu \mathrm{m}$ grains. These twins contained a high degree of disorder as shown in Fig. 5b. Dislocation half-loops are seen emerging from the twin boundaries, and weak-beam dark-field images (not shown) reveal a high density of dislocations within the boundaries. The dislocation density elsewhere in the specimen is low. Following testing, the evolution of the microstructure to the apparent yield point is shown in fig. $5 \mathrm{c}(\mathrm{e}=0.001 \%)$ and $5 \mathrm{~d}(\mathrm{e}=0.01 \%)$. High densities of dislocations now exist within and outside the annealing twins and some twin boundaries appear to have been penetrated by dislocations, Fig 5d. These observations imply that the annealing twin boundaries were sources of partial and perfect dislocations; a similar suggestion has been made by others. ${ }^{\left[7,{ }^{34]}\right.}$ To validate this suggestion, in situ straining experiments were performed using preloaded specimens. This study showed that perfect and partial dislocations are emitted from the annealing twins, that the annealing twins can act as barriers to slip and in other cases slip is transmitted across the twin boundary, that deformation twins are produced and annihilated during the straining process, and, 

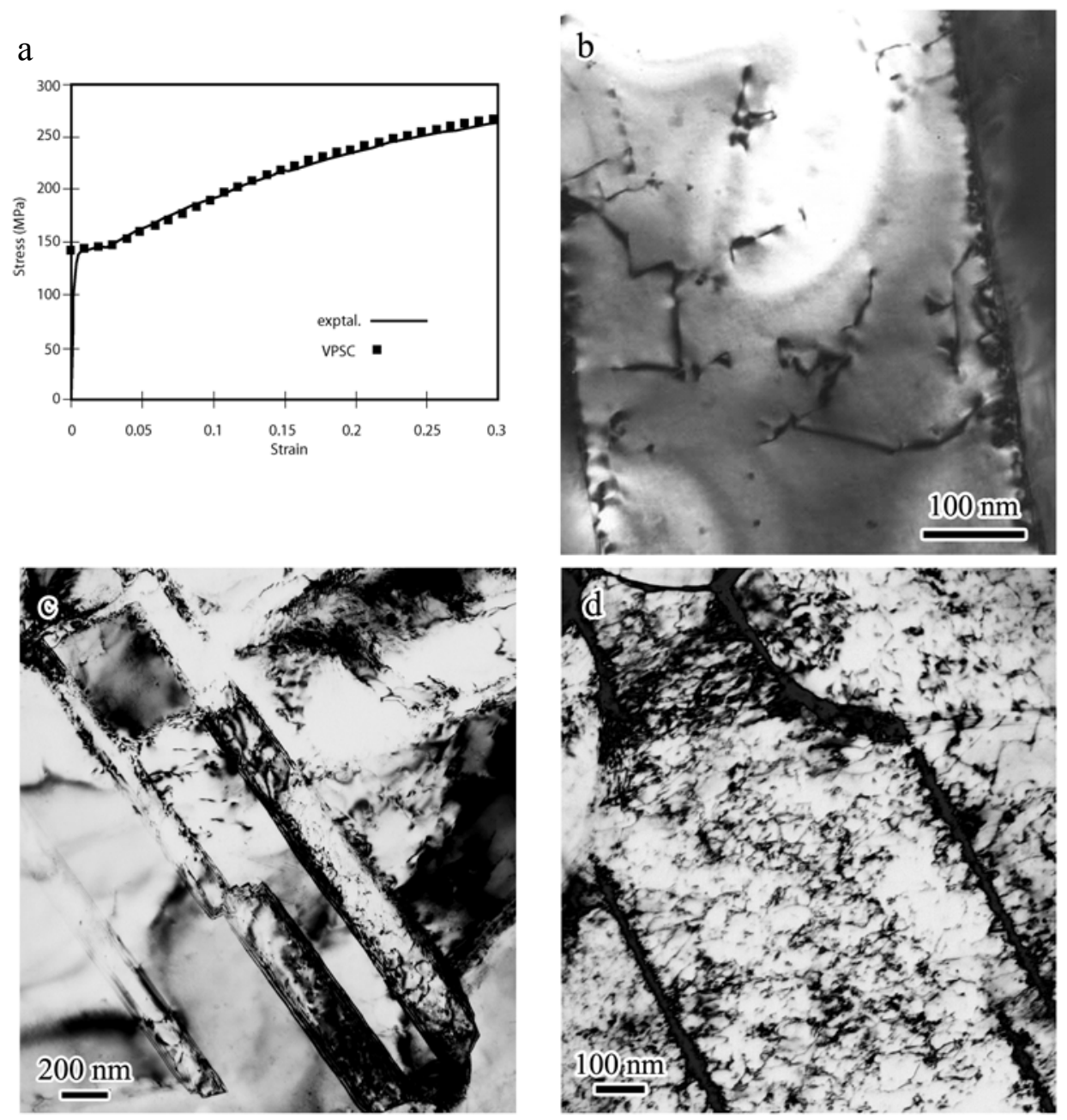

Figure 5. a). stress strain curve of fine grained material, experimental and model; b). initial microstructure; c). microstructure at strain of $0.001 \%$ and d). microstructure at strain of $0.01 \%{ }^{[9]}$

lastly, that annealing twins can be destroyed through the interaction with perfect and partial dislocations. ${ }^{[9]}$

To model the dislocation source generation from the annealing twin boundaries it is necessary to consider the evolution of both mobile, $\rho_{\mathrm{m}}$, and forest, $\rho_{\text {for }}$, dislocation densities during deformation. That is, two internal variables are used; a similar approach has been used to study deformation in metals that involves rapid changes of deformation path. ${ }^{[35,36]}$ Following Kubin and Estrin, ${ }^{[37]}$ the evolution of the mobile and forest dislocation densities can be expressed as:

$$
\begin{gathered}
\dot{\rho}_{m}=\left(k_{1}-k_{2} \rho_{m}-k_{3} \sqrt{\rho_{f}}\right) \sum_{\alpha}\left|\dot{\gamma}^{\alpha}\right| \\
\text { and } \dot{\rho}_{f}=\left(k_{2} \rho_{m}+k_{3} \sqrt{\rho_{f}}-k_{4} \rho_{f}\right) \sum_{\alpha}\left|\dot{\gamma}^{\alpha}\right|
\end{gathered}
$$


In these equations, $\mathrm{k}_{1}, \mathrm{k}_{2}, \mathrm{k}_{3}$ and $\mathrm{k}_{4}$ represent the generation of mobile dislocations, the annihilation and trapping of mobile dislocations, the immobilization of dislocations as they interact with forest dislocations, and dynamic recovery, respectively.

These equations are introduced into a composite grain model in which each composite consists of two phases separated by a $\Sigma 3$ twin boundary. Each composite grain is treated as a viscoplastic ellipsoid embedded in a Homogeneous Equivalent Medium, HEM. Both individual grains and HEM have uniform properties, where the latter represents the aggregate by averaging the response of all composite grains. The viscoplastic self-consistent scheme of Lebensohn and Tomé $^{[38]}$ is used to solve for the heterogeneous plasticity response for given boundary conditions. The macroscopic response is characterized by small dislocation trapping $k_{2}$ and immobility $k_{3}$ coefficients, and large generation $k_{1}$ and annihilation $k_{4}$ coefficients. The results of the model are shown in Fig 5a. The essential feature of the macroscopic response, the elongation of the yield point, is captured by the model.

Conclusions

By combining static and dynamic transmission electron microscopy techniques insight to the dislocation processes responsible for generating the microstructure can be determined. Using the key mechanisms as input to macroscale plasticity models, it is possible to develop models capable of predicting the macroscopic response. In both examples presented in this work, the boundaries, grain and twin, were identified as sources of dislocations.

\section{Acknowledgements}

This work was supported by Lawrence Livermore National Laboratory, the U.S. Department of Energy Stewardship Science Academic Alliances Program, DOE DEFG03-02-NA00072.

and the Center for Simulation of Advanced Rockets under DOE subcontract B341494. Research for this publication was carried out in the Center for Microanalysis of Materials, University of Illinois at Urbana-Champaign, which is partially supported by the U.S. Department of Energy under grant DEFG02-91-ER45439.

\section{References}

1. Beaudoin, A. J., Acharya, A., Chen, S. R., Korzekwa, D. A. and Stout, M. G., Acta Mater. 48 (13), 3409 (2000).

2. Acharya, A., Bassani, J. L. and Beaudoin, A., Scripta Mater. 48 (2), 167 (2003).

3. Devincre, B., Veyssiere, P. and Saada, G., Phil. Mag. A 79 (7), 1609 (1999).

4. Zbib, H. M. and Diaz de la Rubia, T., Inter. J. of Plasticity 18 (9), 1133 (2002).

5. Needleman, A., Acta Mater. 48 (1), 105 (2000).

6. Robach, J. S., Robertson, I. M., Wirth, B. D. and Arsenlis, A., Phil. Mag. 83 (8), 955 (2003).

7. Flinn, J. E., Field, D. P., Korth, G. E., Lillo, T. M. and Macheret, J., Acta Mater. 49 (11), 2065 (2001).

8. Robertson, I. M., Robach, J., Wirth, B. and Arsenlis, A., Multiscale Phenomena in Materials - Experiments and Modeling Related to Mechanical Behavior, Materials Research Society, Pittsburgh, 2003 779,15. 
9. AL-Fadhalah, K., Li, C. M., Beaudoin, A. J. and Robertson, I. M., to be submitted to Acta Materialia, (2004).

10. Allen, C. W. and Ryan, E. A., Microstructure Evolution During Irradiation. Mater. Res. Soc, Pittsburgh, 1997,277.

11. Korzekwa, D. A., Los Alamos National Laboratory supplied the material in this condition.

12. Lyles, R. L., Jr., Rothman, S. J. and Jager, W., Metallography 11 (3), 361 (1978).

13. Victoria, M., Baluc, N., Bailat, C., Dai, Y., Luppo, M. I., Schaublin, R. and Singh, B. N., J. Nucl. Mater. 276, 114 (2000).

14. Singh, B. N., Edwards, D. J. and Toft, P., J. Nucl. Mater. 238 (2-3), 244 (1996).

15. Singh, B. N., Horsewell, A., Toft, P. and Edwards, D. J., J. Nucl. Mater. 224 (2), 131 (1995).

16. Shinohara, K., Yasuda, M., Yasuda, K. and Kutsuwada, M., J. Nucl. Mater. 182, 145 (1991).

17. Himbeault, D. D., Chow, C. K. and Puls, M. P., Metall. Mater.Trans. A: 25A (1), 135 (1994).

18. Edwards, D. J. and Singh, B. N., Proceedings of the 11th Conference on Fusion Research, Dec 7-12 2003, Eds., Kyoto, Japan,Elsevier, Amsterdam, Netherlands, 2004 329-333,1072.

19. Dai, Y., Jia, X., Chen, J. C., Sommer, W. F., Victoria, M. and Bauer, G. S., 4th International Workshop on Spallation Materials Technology, Oct 8-13 2000, Eds., Schruns,Elsevier Science B.V., 2001 296,174.

20. Muller, G. V., Gavillet, D., Victoria, M. and Martin, J. L., J. Nucl. Mater. 212-1 (pt B), 1283 (1994).

21. Eyre, B. L. and Bartlett, A. F., Phil. Mag 11, 53 (1965).

22. Sharp, J. V., Acta Metal. 22 (4), 449 (1974).

23. Trinkaus, H., Singh, B. N. and Foreman, A. J. E., J. Nucl. Mater. 206 (2-3), 200 (1993).

24. Singh, B. N., Foreman, A. J. E. and Trinkaus, H., J. Nucl. Mater. 249 (2-3), 103 (1997).

25. Trinkaus, H., Singh, B. N. and Foreman, A. J. E., J. Nucl. Mater. 251, 172 (1997).

26. Trinkaus, H., Singh, B. N. and Foreman, A. J. E., J. Nucl. Mater. 249 (2-3), 91 (1997).

27. Ghoniem, N. M., Tong, S.-H., Singh, B. N. and Sun, L. Z., Phil. Mag. A 81 (11), 2743 (2001).

28. Singh, B. N., Ghoniem, N. M. and Trinkaus, H., J. Nucl. Mater. 307-311, 159 (2002).

29. Robach, J., Ph. D., University of Illinois, 2004.

30. Hirth, J. P. and Lothe, J., Theory of dislocations. (Wiley, New York, 1982).

31. Daulton, T. L., Kirk, M. A. and Rehn, L. E., J. Nucl. Mater. 276, 258 (2000).

32. Hiratani, M., Zbib, H. M. and Wirth, B. D., Phil. Mag. A 82 (14), 2709 (2002).

33. Arsenlis, A., submitted to Phil. Mag. (2004).

34. Field, D. P., True, B. W., Lillo, T. M. and Flinn, J. E., Mater. Sci. Engn. A 372 (1-2), 173 (2004).

35. Estrin, Y., High Temperature Constitiutive Modelling - Theory and Application, ASME, New York, NY, USA, 1991 26,65.

36. Estrin, Y., Braasch, H. and Brechet, Y., J. Engn. Mater. and Tech. 118 (4), 441 (1996).

37. Kubin, L. P. and Estrin, Y., Acta Metal. 38 (5), 697 (1990).

38. Lebensohn, R. A. and Tomé, C. N., Acta Metall. Mater. 41, 2611-2624. (1993). 